\title{
Educación y brecha digital en tiempos del COVID-19. Perfiles y problemáticas experimentadas por el alumnado juvenil para afrontar sus estudios durante el confinamiento
}

\author{
Education and Digital Divide in Times of COVID-19. \\ Profiles and Problematics Experienced by Young Students \\ to Confront their Studies During the Lockdown
}

\author{
Stribor Kuric Kardelis, Daniel Calderón-Gómez y Anna Sanmartín Ortí
}

\section{Resumen}

En este artículo analizamos los principales perfiles y problemáticas experimentadas por el alumnado de secundaria (obligatoria y postobligatoria) y de estudios universitarios para afrontar el curso académico durante el confinamiento debido a la pandemia del COVID-19. A partir de un análisis cuantitativo bivariado y multivariable, basado en la reciente investigación De puertas adentro y pantallas afuera. Jóvenes en confinamiento (Sanmartín et al., 2020), mostramos cómo los grupos sociales más vulnerables han tenido más dificultades para continuar con la educación online. Además, elaboramos una tipología de dificultades experimentadas por el alumnado (Q1-sin problemas importantes, Q2-problemas técnicos, Q3-problemas psico-sociales, Q4-problemas de competencias) que nos permiten dimensionar los diferentes tipos de dificultades según las características sociodemográficas de los sujetos. La falta de competencias es la problemática más extendida para continuar con los estudios, si bien las condiciones estructurales (falta de tiempo y espacio, acceso a las TIC, estado anímico, etc.) son especialmente relevantes entre los grupos sociales más vulnerables (clase social baja, población extranjera, áreas rurales, mujeres).

\section{Palabras clave}

Educación online, COVID-19, brecha digital, análisis cuantitativo.

\section{Abstract}

In this paper we analyze the main profiles and problematics experienced by secondary (mandatory and non-mandatory) and university students to confront the academic year during the lockdown caused by the COVID-19 pandemic. From a bivariate and multivariate quantitative analysis based on the research De puertas adentro y pantallas afuera. Jóvenes en confinamiento (Sanmartín et al., 2020), we describe how the most vulnerable social groups had difficulties in keeping up with online education. Besides, a typology of difficulties experienced for students it is elaborated (Q1-withouth important problems, Q2-technic problems, Q3-psico-social problems, Q4-competency-problems) to measure the diverse types of difficulties according to subjects' sociodemographic profile. Lack of competencies is the most generalized difficulty to continue studying, although structural conditions (lack of time and space, ICT accessibility, state of mind, etc.) are particularly relevant among the most vulnerable social groups (low-class, foreign population, rural areas, women).

\section{Keywords}

Online education, COVID-19, digital divide, quantitative analysis.

\section{Cómo citar/Citation}

Kuric Kardelis, Stribor; Calderón-Gómez, Daniel y Sanmartín Ortí, Anna (2021). Educación y brecha digital en tiempos del COVID-19. Perfiles y problemáticas experimentadas por el alumnado juvenil para afrontar sus estudios durante el confinamiento. Revista de Sociología de la Educacion-RASE, 14 (1), 63-84. http://dx.doi.org/10.7203/RASE.14.1.18265. 


\section{Introducción}

Es evidente que desde el estallido de la pandemia global nuestros hábitos y prácticas cotidianas se han visto drásticamente alteradas. Esto se observa tanto en un sentido práctico, por la distancia física y el confinamiento, como en términos cognitivo-emocionales, puesto que la percepción colectiva del riesgo ha pasado de la indiferencia y la curiosidad a la preocupación y al miedo en muchos casos (Díez et al., 2020). También las actitudes y expectativas con respecto a la ciencia se han visto trastocadas debido a la sobrecarga de (des)información difundida en Internet (Nguyen y Catalan-Matamoros, 2020), de forma que han emergido importantes sectores negacionistas y teorías de la conspiración que ven en las medidas de confinamiento una forma de control social e intromisión en las libertades individuales. Así, en la reciente encuesta sobre la percepción de los aspectos científicos del COVID realizada por la Federación Española para la Ciencia y la Tecnología (Lobera y Cabrera, 2020), casi un 13,3\% de la población española rechazaría vacunarse inmediatamente contra el coronavirus, si fuera posible, y un 9,8\% se muestran indecisos; únicamente un tercio $(32,2 \%)$ de la población señala, sin ningún atisbo de duda, que se pondría la vacuna con total seguridad.

Por otro lado, la digitalización de las prácticas sociales se ha visto intensificada en una situación de confinamiento y distanciamiento físico entre las personas. Así, aunque es un proceso que se remonta a las últimas décadas del siglo xx (Castells, 2011; Feather, 2013), es evidente que la pandemia nos ha hecho más dependientes de la conectividad proporcionada por las tecnologías digitales e Internet, afectando aspectos de nuestra vida como son el trabajo (teletrabajo), la educación (online), la comunicación (a distancia) o las prácticas de ocio posibilitadas a través de la miríada de dispositivos TIC conectados al ingente repositorio multimedia que constituye Internet. Sin embargo, esta dependencia tecnológica hace emerger nuevos perfiles de riesgo y nuevas formas de vulnerabilidad (Robinson et al., 2020), puesto que la desigualdad digital se convierte cada vez más en un factor central de estratificación social en un contexto en que las TIC se han vuelto esenciales para mantener nuestra actividad social. Como muestra la reciente investigación de Van Deursen (2020) sobre la desigualdad digital durante la pandemia, se está alimentando un círculo vicioso por el cual los grupos más vulnerables están siendo marginalizados aún más en tiempo de crisis. Oltra y Boso (2020) señalan que identificar y ayudar a los grupos vulnerables es una lección fundamental en cualquier crisis y la del COVID-19 no es una excepción.

Es crucial adoptar medidas que puedan paliar las problemáticas de las personas afectadas más significativamente por la crisis para poder salir de la misma. Para ello, planteamos este estudio exploratorio sobre las dificultades experimentadas por el alumnado a la hora de adaptarse a la educación online durante el confinamiento.

\section{Desigualdad digital y educación online en tiempos de confinamiento}

Desde las políticas de inclusión digital (Reisdorf y Rhinesmith, 2020), se entiende que la participación digital de la ciudadanía es un componente fundamental para favorecer la inclusión social. Sin embargo, las barreras al aprovechamiento tecnológico van más allá del mero acceso físico a los dispositivos tecnológicos - la primera brecha digital_, sino que tienen que ver con las condiciones materiales de acceso (Gonzales, 2016; Van Deursen y van Dijk, 2019), las competencias digitales, motivaciones y formas diferenciales de uso — la segunda brecha digital (Castaño, 2008; Hargittai, 2002)—y, más recientemente, con los beneficios offline tangibles que los sujetos obtienen del uso diferencial de las TIC — la tercera brecha digital (Ragnedda, 2017; Van Deursen y Helsper, 2015). 
La manera particular en que las diferentes brechas digitales se articulan y polarizan las desigualdades sociales ha sido especialmente relevante durante el confinamiento provocado por el COVID-19, debido a la necesidad de continuar los programas escolares y académicos a distancia, a través de Internet. Aunque no existe una literatura consolidada, algunos primeros estudios (Expósito y Marsollier, 2020; Sevilla et al., 2020) están planteando que la pérdida de presencialidad supone una quiebra de la igualdad de oportunidades que debe garantizar el sistema educativo, haciendo recaer en las familias una tarea que debería suplir la institución. No es ninguna novedad que la existencia de desigualdades sociales afecta negativamente al desarrollo educativo, no obstante, el confinamiento ha desnudado los defectos de un sistema incapaz de dar una respuesta coordinada a las necesidades específicas de cada familia. Investigaciones como la de Bonal y González (2020) muestran que el capital económico, social y cultural de las familias determinan directamente la capacidad que han tenido los estudiantes para adaptarse al nuevo contexto educativo durante el confinamiento. En otras palabras, la educación online agrava las desigualdades sociales. A los problemas de accesibilidad TIC y conectividad debemos añadir la falta de competencias digitales, el capital cultural general del entorno familiar y la existencia de problemas específicos de convivencia, vulnerabilidad y pobreza que afectan al seguimiento del curso académico. También han cobrado relevancia aspectos relacionados con las desigualdades de género — violencia machista, discriminación, formación en valores e igualdad, etc. (Penna Tosso et al., 2020)—, así como la violencia contra la infancia y la adolescencia (Fundación ANAR, 2020). Este tipo de problemáticas se trataban de forma presencial en los centros educativos se han visto invisibilizadas y desatendidas ante la falta de organización institucional y la sobrecarga de trabajo de profesores y profesionales educativos.

En este sentido, la valoración del profesorado de la gestión del curso académico durante la pandemia ha sido bastante crítica, como muestra la reciente investigación realizada por la Fundación de Ayuda contra la Drogadicción (Trujillo Sáez et al., 2020). Principalmente, se señala el abandono de los centros por parte de las administraciones, mostrándose el profesorado especialmente preocupado por el seguimiento de la actividad del alumnado durante la pandemia, en un contexto donde una enorme variedad de problemas han surgido, entre los que podemos destacar problemas de tipo técnico (acceso a los dispositivos y herramientas tecnológicas necesarias), de tipo cognitivo (carencias de competencias digitales por parte del profesorado, pero también del alumnado) y de tipo psico-social (estado anímico, estrés, ansiedad, incertidumbre, preocupación por el entorno social y demás malestares que afectan al rendimiento académico).

En resumen, aunque la visión del profesorado y profesionales del ámbito educativo sí que ha sido escuchada, aún tenemos una falta de investigaciones centradas en la percepción y experiencias del alumnado durante el confinamiento. Por ello, en este artículo vamos a centrarnos en dimensionar las principales dificultades y problemáticas experimentadas por el alumnado de secundaria y universitario para continuar con sus estudios de manera telemática, con un especial énfasis en las problemáticas derivadas de las diversas brechas digitales que afectan a la población (Ragnedda, 2017) y en relación con otro tipo de circunstancias personales y socio-estructurales que inciden en el desarrollo de la actividad formativa desde casa. Se trata de un estudio exploratorio que busca abrir la puerta a futuras investigaciones más en profundidad sobre este tema, indagando en la influencia de las características sociodemográficas y condiciones sociales del alumnado en el seguimiento de la educación online. 


\section{Metodología}

Este artículo sigue una orientación cuantitativa a partir del uso de la metodología de encuesta, a partir de un cuestionario estructurado a población juvenil (entre 15 y 29 años) sobre educación, teletrabajo, formas de ocio y usos de las tecnologías digitales durante el confinamiento provocado por la COVID-19. Este cuestionario ha sido elaborado por el Centro Reina Sofía sobre Adolescencia y Juventud (CRS) como parte de la investigación De puertas adentro y pantallas afuera. Jóvenes en confinamiento (Sanmartín et al., 2020), dedicada a las experiencias de la juventud durante la pandemia, incorporando preguntas relevantes sobre seguimiento de estudios durante el confinamiento y dificultades vividas en este periodo.

Se trata de un cuestionario online autoadministrado a 1200 jóvenes de entre 15 y 29 años residentes en España. El muestreo incluye cuotas de edad (15-19, 20-24 y 25-29 años) y de nivel de estudios terminados (hasta secundarios obligatorios, secundarios postobligatorios y superiores). El trabajo de campo se realizó en mayo de 2020, justo durante el proceso de desescalada del confinamiento debido a la pandemia de la COVID-19. Para este artículo, del total de 1200 casos nos hemos centrado específicamente en el grupo de jóvenes que están estudiando en la actualidad (731 casos). Asumiendo muestreo aleatorio simple y $\mathrm{p}^{*} \mathrm{q}=0,50$, a un nivel de confianza del 95,5\%, el error muestral es del +-3,6\%. Las características de la muestra se incluyen en la Tabla I.

Tabla I. Características sociodemográficas de la muestra

\begin{tabular}{|c|c|c|c|}
\hline & & $\mathbf{N}$ & $\%$ \\
\hline TOTAL & & 731 & 100 \\
\hline \multirow{3}{*}{ Género } & Mujeres & 364 & 49,8 \\
\hline & Hombres & 365 & 49,9 \\
\hline & Otro género & 2 & 0,3 \\
\hline \multirow{3}{*}{ Edad } & 15 a 19 años & 310 & 42,4 \\
\hline & 20 a 24 años & 255 & 34,9 \\
\hline & 25 a 29 años & 166 & 22,7 \\
\hline \multirow{3}{*}{ Clase social } & Alta y Media-Alta & 146 & 20,3 \\
\hline & Media & 396 & 55 \\
\hline & Baja y Media Baja & 178 & 24,7 \\
\hline \multirow{2}{*}{ Estudios (actualidad) } & Educación secundaria & 327 & 45,7 \\
\hline & Educación universitaria & 388 & 54,3 \\
\hline \multirow{3}{*}{ Situación laboral } & Estudia y trabaja & 336 & 46 \\
\hline & Únicamente estudia & 274 & 37,5 \\
\hline & Estudia y busca trabajo & 121 & 16,6 \\
\hline \multirow{3}{*}{ Nacionalidad } & Española de nacimiento & 612 & 83,7 \\
\hline & Española adquirida & 66 & 9 \\
\hline & Otra nacionalidad & 53 & 7,3 \\
\hline \multirow{3}{*}{ Hábitat } & 10000 habitantes o menos & 188 & 26,1 \\
\hline & Entre 10000 h. y 1 millón & 393 & 54,5 \\
\hline & Un millón de h. y más & 140 & 19,4 \\
\hline \multirow{4}{*}{ Convivencia } & Solo/a & 47 & 6,5 \\
\hline & Con sus padres & 498 & 68,5 \\
\hline & Con su pareja & 134 & 18,4 \\
\hline & Comparte piso & 48 & 6,6 \\
\hline \multirow{3}{*}{ Ideología } & Izquierda & 197 & 30,1 \\
\hline & Centro & 312 & 47,6 \\
\hline & Derecha & 146 & 22,3 \\
\hline
\end{tabular}

Fuente: de puertas adentro y pantallas afuera. Jóvenes en confinamiento (Centro Reina Sofía sobre adolescencia y juventud). 
Con respecto a la explotación estadística, se han utilizado técnicas univariadas (frecuencias relativas), bivariadas (análisis de tablas de contingencia y diferencias significativas), así como multivariantes (análisis factorial de componentes principales, análisis de conglomerados K-medias y análisis discriminante). Con respecto a los análisis bivariados, se han realizado cruces por género, grupos quinquenales de edad, nivel de estudios terminado y actual, clase social auto-percibida (alta, media alta, media, media baja, baja), compaginación de estudios y trabajo, nacionalidad, tipo de hábitat residencial, situación de convivencia e ideología.

El modelo multivariable planteado parte de la implementación sucesiva de análisis factorial, análisis de conglomerados y análisis discriminante. En primer lugar, se ha utilizado un análisis factorial de componentes principales para agrupar 18 preguntas sobre dificultades y problemas experimentados por el alumnado en la educación online durante la pandemia en 8 factores latentes. Como criterio de convergencia, se ha priorizado la interpretabilidad social de los factores, alcanzando un total de varianza explicada del $57 \%$ y aplicando una rotación de tipo ortogonal (Varimax). El N total es de 656 casos, ya que se excluyen a aquellos jóvenes que no han podido continuar sus estudios durante el confinamiento.

Tabla II. FA2. Formas de uso de Internet. Matriz componentes rotados

\begin{tabular}{|c|c|c|c|c|c|c|c|c|}
\hline \multirow[t]{2}{*}{ VARIABLES EN EL MODELO } & \multicolumn{8}{|c|}{ COMPONENTE } \\
\hline & F1 & F2 & F3 & F4 & F5 & F6 & F7 & F8 \\
\hline Varianza explicada & $8,7 \%$ & $7,7 \%$ & $7,4 \%$ & $6,9 \%$ & $6,8 \%$ & $6,7 \%$ & $6,6 \%$ & $6,3 \%$ \\
\hline El profesorado no estaba lo suficientemente pendiente & $0,71^{\star}$ & $-0,07$ & $-0,10$ & 0,11 & 0,07 & $-0,02$ & $-0,04$ & $-0,07$ \\
\hline El profesorado no estaba bien preparado para impartir clases online & $0,66^{*}$ & 0,12 & 0,23 & $-0,05$ & $-0,05$ & 0,01 & 0,04 & $-0,10$ \\
\hline No estaban claras mis tareas & $0,63^{*}$ & 0,00 & 0,01 & $-0,04$ & 0,04 & 0,12 & $-0,07$ & 0,22 \\
\hline En casa no tengo un espacio adecuado para estudiar & 0,01 & $0,69 *$ & $-0,11$ & 0,04 & $-0,12$ & 0,14 & 0,28 & 0,00 \\
\hline No encuentro tranquilidad en casa por las personas con quienes vivo & $-0,14$ & $0,62^{*}$ & 0,10 & $-0,03$ & 0,22 & 0,24 & $-0,10$ & 0,16 \\
\hline En casa no me concentro & 0,23 & $0,58^{\star}$ & 0,19 & 0,05 & 0,11 & $-0,30$ & $-0,06$ & $-0,04$ \\
\hline Me resulta difíil seguir las clases o tutorías online & 0,01 & 0,14 & $0,71^{*}$ & 0,15 & 0,01 & $-0,08$ & $-0,11$ & 0,01 \\
\hline Tenía dudas que no sabía resolver por mi cuenta & 0,24 & $-0,19$ & $0,50^{*}$ & 0,02 & 0,05 & 0,26 & 0,02 & 0,44 \\
\hline La conexión a internet no es buena & $-0,07$ & 0,09 & 0,20 & $0,69 *$ & 0,12 & $-0,18$ & 0,19 & 0,15 \\
\hline El equipo o la conexión del profesorado no era buena & 0,15 & $-0,16$ & 0,22 & $0,60^{*}$ & $-0,03$ & 0,25 & 0,11 & $-0,26$ \\
\hline No sé cómo utilizar las herramientas/aplicaciones online que utilizan mis profesores/as & 0,01 & 0,12 & $-0,35$ & $0,58 *$ & $-0,08$ & 0,12 & $-0,25$ & 0,08 \\
\hline No me he encontrado bien anímicamente & 0,11 & 0,15 & 0,14 & 0,01 & $0,80^{*}$ & $-0,14$ & $-0,12$ & $-0,05$ \\
\hline He tenido problemas de salud & $-0,04$ & $-0,06$ & $-0,16$ & 0,04 & $0,67^{\star}$ & 0,24 & 0,36 & 0,03 \\
\hline El profesorado tenía que atender a demasiados alumnos/as & 0,07 & 0,10 & $-0,06$ & 0,11 & 0,02 & $0,67^{*}$ & $-0,14$ & 0,06 \\
\hline Hay partes de mis estudios que no se pueden hacer en casa & 0,09 & 0,05 & 0,42 & $-0,10$ & 0,01 & $0,49 *$ & 0,18 & $-0,40$ \\
\hline Me resulta difícil utilizar el ordenador & 0,07 & $-0,04$ & $-0,13$ & 0,02 & $-0,04$ & $-0,08$ & $0,68^{\star}$ & 0,01 \\
\hline He tenido que cuidar de otras personas & $-0,15$ & 0,15 & 0,09 & 0,07 & 0,12 & $-0,03$ & $0,53^{\star}$ & 0,09 \\
\hline No tengo ordenador propio & 0,01 & 0,10 & 0,03 & 0,01 & $-0,03$ & 0,00 & 0,12 & $0,76^{*}$ \\
\hline
\end{tabular}

Datos: puntuaciones factoriales/*Puntuación más elevada de la variable y un factor.

En la Tabla II se resumen las puntuaciones factoriales rotadas de los factores, cuyas características se enumeran a continuación:

- F1. Profesorado. Agrupa variables relacionadas con la percepción juvenil sobre el interés, la preparación y la organización de las clases por parte del profesorado. 
- F2. Falta de espacio. Tiene que ver con la imposibilidad de disponer de un espacio propio y de tranquilidad en el hogar para poder seguir las clases, así como la falta de concentración.

- F3. Falta de competencias personales. Dificultades de seguimiento de las clases online y de resolución de dudas o problemas a través de internet.

- F4. Problemas técnicos. Problemas de conexión, fallos en los equipos y de competencias digitales necesarias para utilizar las plataformas y herramientas necesarias para seguir las clases.

- F5. Estado anímico. Problemas de salud y relacionados con el estado de ánimo durante la pandemia.

- F6. Problemas organizativos. Ratio de alumnos por profesor demasiado elevada y dificultades para realizar determinadas tareas de manera telemática.

- F7. Falta de tiempo. Falta de tiempo para estudiar y tener a otras personas a su cargo.

- F8. No tiene ordenador. No dispone de ordenador para poder seguir las clases online.

A partir de los 8 factores de dificultades experimentadas durante el confinamiento y del índice estandarizado del número de dificultades mencionadas se ha realizado una tipología que ha dado como resultado 4 grupos de jóvenes en función del tipo de problemáticas vividas. Para ello, se ha utilizado un análisis de conglomerados K-medias, una técnica de interdependencia que permite agrupar los casos en base a una serie de variables de escala (8 factores más el índice estandarizado de dificultades). Todas las variables introducidas son estadísticamente significativas al 95,5\%, siendo la distribución de casos en los grupos la siguiente:

- Q1. Sin problemas importantes: 349 casos $(53,2 \%)$

- Q2. Problemas técnicos: 34 casos (5,2\%)

- Q3. Problemas psicosociales: 56 casos $(8,5 \%)$

- Q4. Problemas de competencias: 217 casos (33,1\%).

Finalmente, como método de comprobación de la adecuación de la tipología construida, se ha realizado un análisis discriminante. Este análisis de dependencia es habitual como complemento confirmatorio al análisis K-medias (Cea D'Ancona, 2016). Se ha obtenido un modelo Lamda de Wilks (significativo al 95,5\%) con tres funciones discriminantes, si bien centraremos el análisis en las dos primeras (FD1 y FD2), que acumulan una varianza del $83,1 \%$.

- FD1. Discrimina los casos principalmente a partir del índice promedio de dificultades mencionadas. Acumula un 54,1\% de varianza y una correlación canónica de 0,908.

- FD2. Discrimina según las dificultades de falta de tiempo, estado anímico, problemas de competencias y problemas del profesorado, acumulando un $29 \%$ de varianza y una correlación canónica de 0,846 .

- FD3. Discrimina según los problemas técnicos y de competencias del alumnado, acumulando un $16,9 \%$ de varianza y una correlación canónica de 0,771 . 


\section{Resultados principales}

En esta sección se describen los principales resultados del análisis estadístico realizado, comenzando por la descripción de los principales indicadores univariados y bivariados para finalizar con la presentación de la tipología de dificultades de seguimiento de los estudios durante la pandemia.

\subsection{El seguimiento de la educación conectada}

Como punto de partida, entre el alumnado de 15 a 29 años el seguimiento de los estudios (Gráfico 1) durante el confinamiento ha sido muy amplio (casi del 90\%), si bien un 8,9\% de jóvenes entrevistados señala no haber continuado sus estudios durante la pandemia, representando dinámicas de abandono escolar y universitario. Por perfil sociodemográfico encontramos algunas diferencias significativas por nacionalidad y situación de convivencia: el seguimiento de la educación online ha sido algo menor entre jóvenes con nacionalidad española adquirida (80,3\%) y de nacionalidad extranjera $(79,2 \%)$, en comparación con la población española de nacimiento (91,7\%). Asimismo, el seguimiento ha sido también ligeramente superior entre quienes viven con sus padres (92\%) frente a otras situaciones de convivencia. Con respecto al resto de variables, si bien no pueden asegurarse diferencias significativas, en los datos se presenta un seguimiento menor a partir de los 20 años $(88,6 \%$ entre 20 y 24 años y $88,4 \%$ entre los 25 y 29 años), entre los hombres (88,6\%), en comparación con las mujeres (90,7\%) y entre las personas de clase baja-media baja $(85,4 \%)$.

\section{Gráfico 1. Seguimiento de los estudios durante el confinamiento}

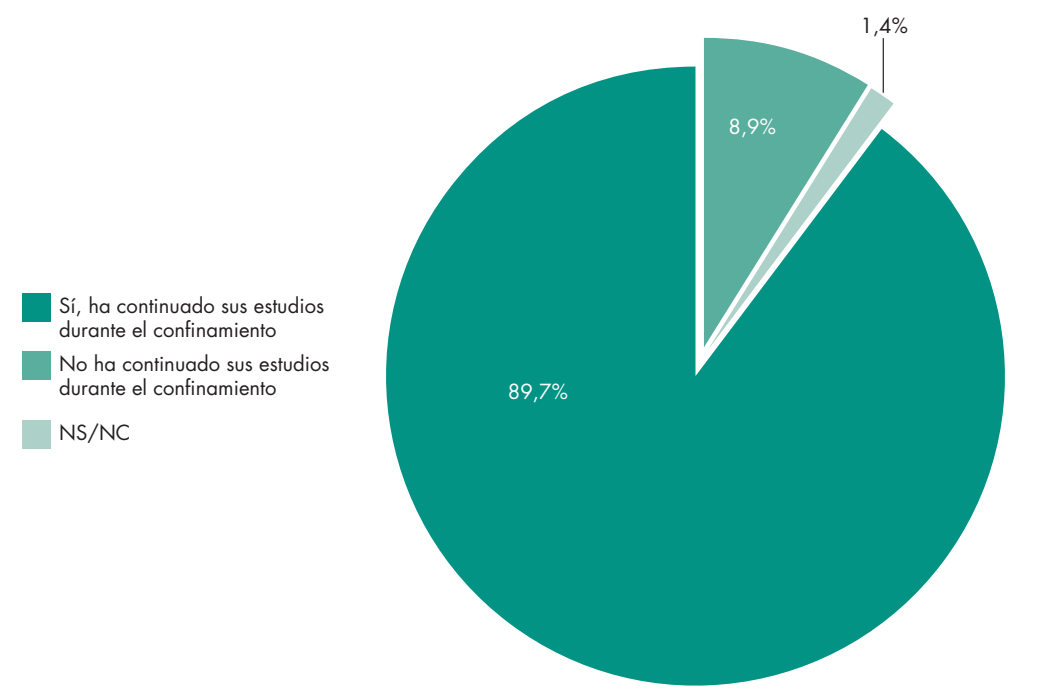

Base: jóvenes entre 15 y 29 años que estudian en la actualidad ( $N=731$ casos).

Centrándonos en el grupo de jóvenes que han podido continuar con sus estudios durante la pandemia $^{2}$, en el Gráfico 2 vemos cómo este seguimiento del curso académico no ha estado exento de dificultades, pues únicamente algo más de un cuarto de estudiantes destaca haber seguido las clases perfectamente, mientras que un $71 \%$ ha experimentado dificultades.

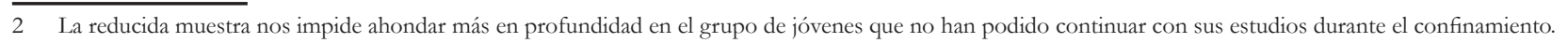




\section{Gráfico 2. Dificultades para continuar los estudios}

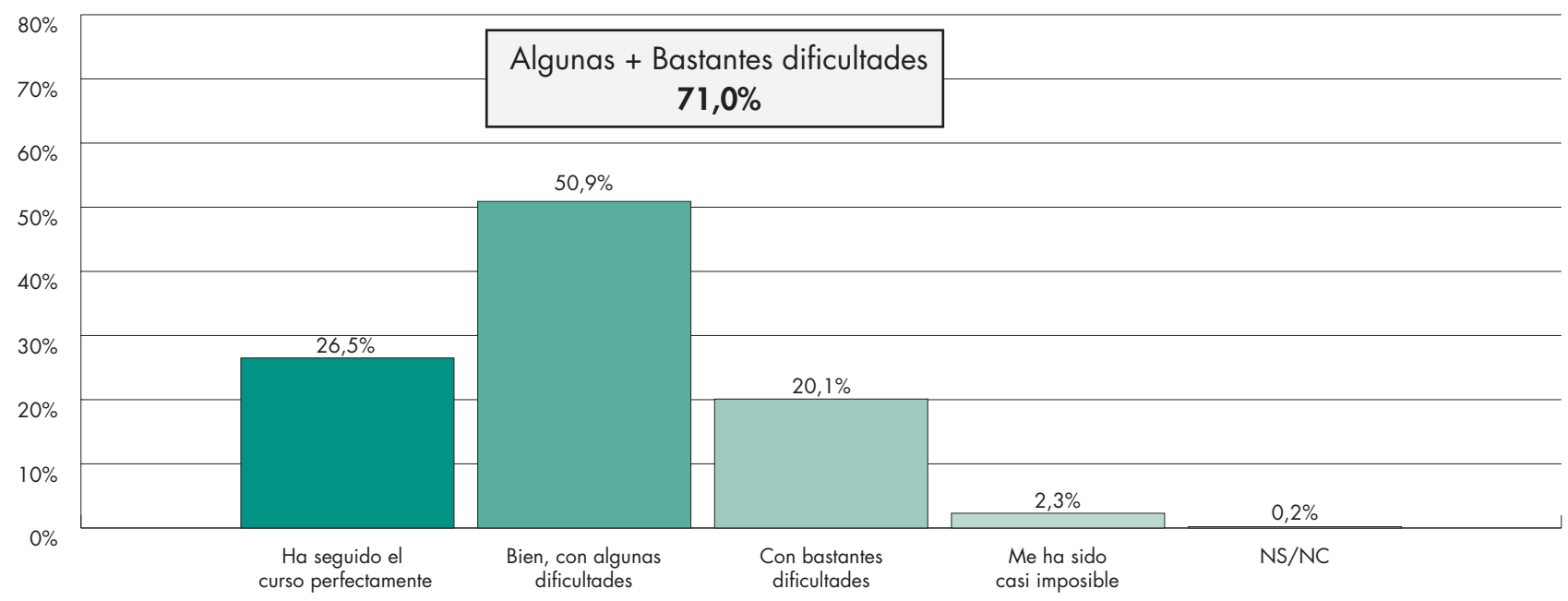

Base: jóvenes entre 15 y 29 años que han podido continuar sus estudios durante el confinamiento ( $\mathrm{N}=656$ casos).

De nuevo encontramos importantes diferencias significativas en la vivencia de dificultades para continuar los estudios, en este caso por clase social subjetiva. Así, el seguimiento del curso sin dificultades (perfectamente) presenta 15 puntos de diferencia entre el alumnado de clase alta-media alta $(36,4 \%)$ y de clase baja-media baja $(21,7 \%)$. Además, más de un cuarto de jóvenes de clase baja y media baja $(27 \%)$ ha tenido bastantes dificultades para continuar sus estudios. Sin marcar diferencias significativas, la vivencia de dificultades en los estudios durante la pandemia también es ligeramente mayor entre las mujeres (el 75,6 han tenido algunas o bastantes dificultades, frente al 66,3\% de los hombres) y entre quienes cursan estudios universitarios (74,4\% frente al $66,4 \%$ de quienes cursan estudios secundarios).

\section{Gráfico 3. Consecuencias del confinamiento}

El confinamiento ha afectado sus estudios PARA BIEN

El confinamiento ha afectado sus estudios PARA MAL

El confinamiento NO ha afectado a sus estudios

NS/NC

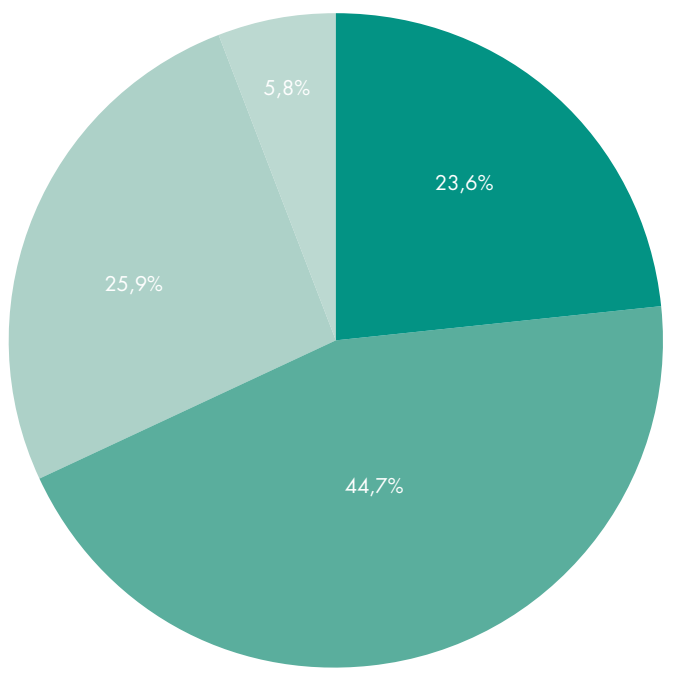

Base: jóvenes entre 15 y 29 años que han podido continuar sus estudios durante el confinamiento ( $\mathrm{N}=656$ casos). 
Los problemas y dificultades en la continuación de la actividad académica se traducen en el hecho de que casi la mitad de los alumnos (44,7\%) destacan que el confinamiento ha afectado a sus estudios negativamente (Gráfico 3). En esta afección negativa al rendimiento académico destacan los jóvenes de clase baja-media baja (49,3\%) y clase media (45,1\%). Además, el efecto negativo es más habitual entre quienes cursan estudios universitarios (49,4\%). Sin ser significativa estadísticamente, sí que es interesante que la afección negativa también es más habitual entre las mujeres (46,8\% frente al 42,7\% de los hombres) y jóvenes entre 20 y 24 años $(51,3 \%)$.

\section{Gráfico 4. Cumplimiento de objetivos académicos}

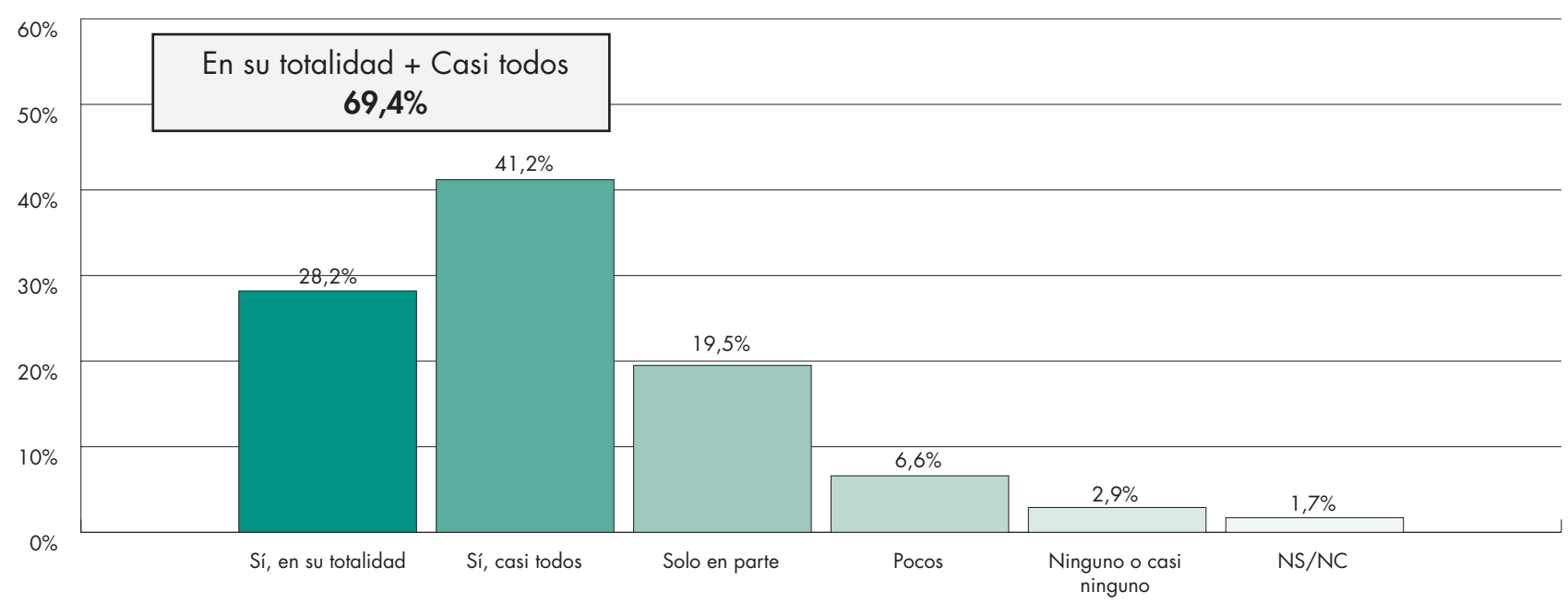

Base: jóvenes entre 15 y 29 años que han podido continuar sus estudios durante el confinamiento ( $\mathrm{N}=656$ casos).

A pesar de las dificultades experimentadas, más de dos tercios $(69,4 \%)$ han podido cumplir la mayor parte de sus objetivos académicos durante el confinamiento (Gráfico 4), si bien únicamente el $28,2 \%$ de alumnos han cumplido absolutamente todos los objetivos. En el caso del cumplimiento de objetivos académicos únicamente encontramos diferencias significativas por nivel académico cursado: así, la resolución de todos los objetivos académicos es más frecuente en la educación secundaria $(32,9 \%)$ que en la universitaria (23,9\%). Sin ser significativa estadísticamente, también es relevante la diferencia que encontramos por clase social, puesto que un 40,3\% de jóvenes de clase alta-media alta, frente a un $24,3 \%$ de clase media y un $26,3 \%$ de clase baja-media baja, han cumplido todos sus objetivos académicos. 


\subsection{Tipología de dificultades para continuar los estudios de forma telemática}

\section{Gráfico 5. Tipo de dificultades experimentadas}

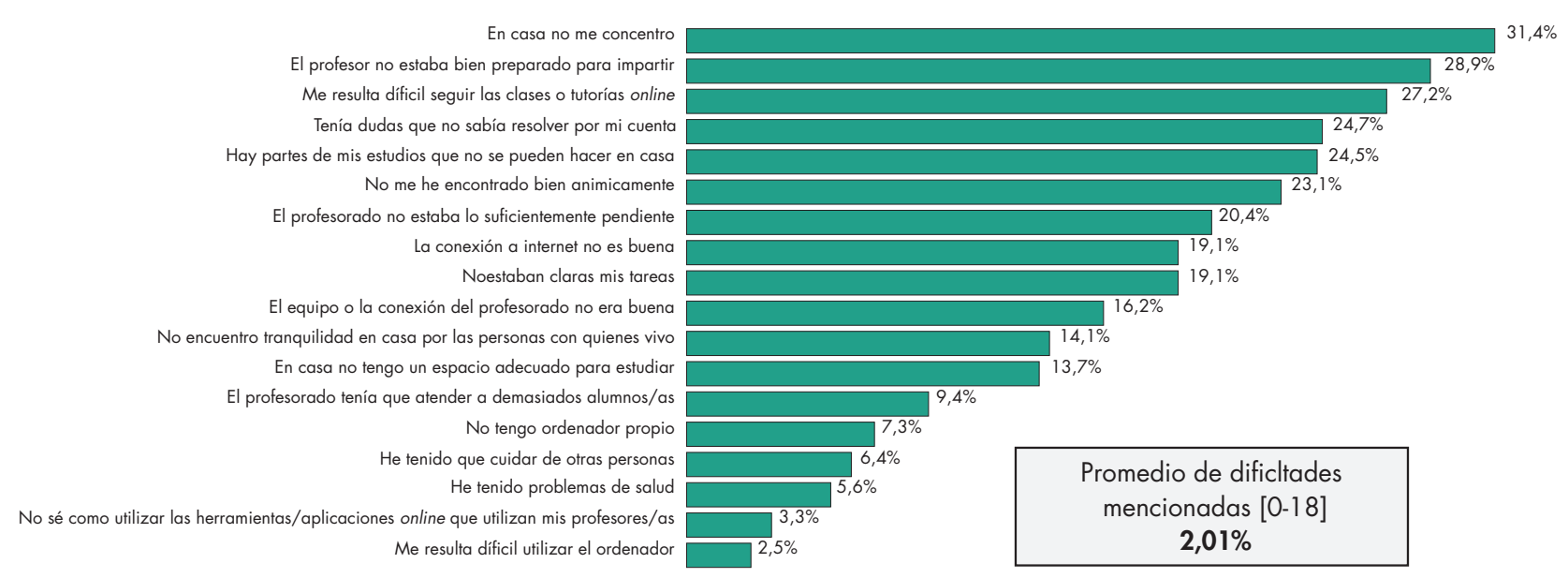

Base: jóvenes entre 15 y 29 años que han podido continuar sus estudios durante el confinamiento (N=656 casos).

Si atendemos al conjunto de dificultades incluido en el cuestionario (Gráfico 5), vemos una gran dispersión y distribución de respuestas entre la juventud, con un promedio de 2,01 dificultades mencionadas por los entrevistados (de un total de 18). Aunque no alcanzan menciones por encima de un tercio del alumnado, los problemas más habituales tienen que ver con la falta de concentración $(31,4 \%)$, la preparación del profesorado $(28,9 \%)$ y las dificultades para seguir las clases $(27,2 \%)$, mientras que son muy poco frecuentes los problemas vinculados con el acceso técnico a los dispositivos tecnológicos o el desconocimiento de cómo usarlos, así como los problemas de salud o tener que cuidar a otras personas. También en esta variable encontramos diferencias significativas por género, edad, nivel de estudios cursado, situación de convivencia e ideología. Así, el promedio de dificultades mencionadas es mayor entre las mujeres $(2,20)$, los jóvenes entre 20 y 24 años $(2,12)$, quienes cursan estudios universitarios $(2,28)$, quienes conviven con sus padres $(2,15)$ y las personas de ideología de izquierdas $(2,42)$. Sin presentar diferencias significativas, el promedio de dificultades también es mayor entre la clase baja-media baja $(2,16)$.

Para profundizar en la agrupación y relación entre las distintas dificultades, presentamos a continuación los resultados de la tipología construida a partir del análisis K-medias: en la Tabla III se muestran las principales puntuaciones de cada conglomerado y en la Tabla IV se recogen sus características sociodemográficas y de seguimiento del curso académico durante la pandemia. 
Tabla III. Análisis de conglomerados K-medias. Resumen del modelo

\begin{tabular}{|c|c|c|c|c|}
\hline \multirow{2}{*}{ VARIABLES EN EL MODELO } & \multicolumn{4}{|c|}{ CONGLOMERADOS } \\
\hline & $\begin{array}{c}\text { Q1. } \\
\text { SIN } \\
\text { PROBLEMAS }\end{array}$ & $\begin{array}{c}\text { Q2. } \\
\text { PROBLEMAS } \\
\text { TÉCNICOS }\end{array}$ & $\begin{array}{c}\text { Q3. } \\
\text { PROBLEMAS } \\
\text { PSICOSOCIALES }\end{array}$ & $\begin{array}{c}\text { Q4. } \\
\text { PROBLEMAS } \\
\text { COMPETENCIAS }\end{array}$ \\
\hline Número de dificultades mencionadas (estandarizada) & $-0,336$ & 1,522 & 1,548 & 1,600 \\
\hline F1. Profesorado & $-0,436$ & 0,042 & $-0,241$ & 0,757 \\
\hline F2. Falta de espacio & $-0,344$ & 0,490 & 0,237 & 0,415 \\
\hline F3. Falta competencias personales & $-0,354$ & $-0,729$ & $-0,192$ & 0,734 \\
\hline F4. Problemas técnicos & $-0,317$ & 3,098 & 0,259 & $-0,042$ \\
\hline F5. Estado anímico & $-0,275$ & $-0,428$ & 1,390 & 0,151 \\
\hline F6. Problemas organizativos & $-0,224$ & 0,729 & 0,305 & 0,168 \\
\hline F7. Falta de tiempo & $-0,184$ & $-0,562$ & 2,396 & $-0,234$ \\
\hline F8. No tiene ordenador & $-0,098$ & 0,138 & 0,246 & 0,073 \\
\hline Número de casos y porcentaje sobre el total & $349(53,2 \%)$ & $34(5,2 \%)$ & $56(8,5 \%)$ & $217(33,1 \%)$ \\
\hline
\end{tabular}

Datos: centros finales de los conglomerados.

Tabla IV. Análisis de conglomerados K-medas. Perfil sociodemográfico y seguimiento del curso

\begin{tabular}{|c|c|c|c|c|c|c|}
\hline VARIABLES & N EL MODELO & $\begin{array}{c}\text { TOTAL } \\
\text { MUESTRA }\end{array}$ & $\begin{array}{c}\text { Q1. } \\
\text { SIN } \\
\text { PROBLEMAS }\end{array}$ & $\begin{array}{c}\text { Q2. } \\
\text { PROBLEMAS } \\
\text { TÉCNICOS }\end{array}$ & $\begin{array}{c}\text { Q3. } \\
\text { PROBLEMAS } \\
\text { PSICOSOCIALES }\end{array}$ & $\begin{array}{c}\text { Q4. } \\
\text { PROBLEMAS } \\
\text { COMPETENCIAS }\end{array}$ \\
\hline \multirow{2}{*}{ Sexo } & Mujeres & 50,1 & 47,4 & 47,1 & 57,1 & 54,6 \\
\hline & Hombres & 49,9 & 52,6 & 52,9 & 42,9 & 45,4 \\
\hline \multirow{3}{*}{ Edad* } & 15 a 19 años & 42,4 & 46,1 & 44,1 & 30,4 & 43,3 \\
\hline & 20 a 24 años & 34,9 & 31,5 & 44,1 & 32,1 & 38,2 \\
\hline & 25 a 29 años & 22,7 & 22,3 & 11,8 & 37,5 & 18,4 \\
\hline \multirow{3}{*}{ Clase social* } & Alta y Media-Alta & 20,3 & 22,1 & 21,2 & 23,2 & 15,4 \\
\hline & Media & 55,0 & 57,3 & 69,7 & 39,3 & 57,9 \\
\hline & Baja y Media Baja & 24,7 & 20,6 & 9,1 & 37,5 & 26,6 \\
\hline \multirow{2}{*}{ Estudios que cursa } & Educación secundaria & 45,7 & 52,5 & 41,2 & 44,4 & 34,9 \\
\hline & Educación universitaria & 54,3 & 47,5 & 58,8 & 55,6 & 65,1 \\
\hline \multirow{3}{*}{ Situación laboral } & Estudia y trabaja & 46,0 & 43,8 & 44,1 & 60,7 & 45,2 \\
\hline & Únicamente estudia & 37,5 & 40,7 & 35,3 & 17,9 & 40,6 \\
\hline & Estudia y busca trabajo & 16,6 & 15,5 & 20,6 & 21,4 & 14,3 \\
\hline \multirow{3}{*}{ Nacionalidad } & Española de nacimiento & 83,7 & 84,0 & 91,2 & 82,1 & 88,0 \\
\hline & Española adquirida & 9,0 & 8,9 & 2,9 & 8,9 & 7,4 \\
\hline & Otra nacionalidad & 7,3 & 7,2 & 5,9 & 8,9 & 4,6 \\
\hline \multirow{3}{*}{ Hábitat } & 10000 habitantes o menos & 26,1 & 25,2 & 29,4 & 37,5 & 24,7 \\
\hline & Entre 10000 h. y 1 millón & 54,5 & 56,5 & 52,9 & 44,6 & 55,3 \\
\hline & Un millón de h. y más & 19,4 & 18,3 & 17,6 & 17,9 & 20,0 \\
\hline \multirow{4}{*}{ Convivencia* } & Solo/a & 6,5 & 6,0 & 5,9 & 5,4 & 6,5 \\
\hline & Con sus padres & 68,5 & 67,2 & 76,5 & 53,6 & 78,1 \\
\hline & Con su pareja & 18,4 & 19,3 & 14,7 & 35,7 & 10,2 \\
\hline & Comparte piso & 6,6 & 7,5 & 2,9 & 5,4 & 5,1 \\
\hline \multirow{3}{*}{ Ideología } & Izquierda & 30,1 & 23,8 & 25,0 & 21,2 & 39,9 \\
\hline & Centro & 47,6 & 49,3 & 50,0 & 61,5 & 44,2 \\
\hline & Derecha & 22,3 & 26,8 & 25,0 & 17,3 & 15,9 \\
\hline
\end{tabular}




\begin{tabular}{|c|c|c|c|c|c|c|}
\hline \multicolumn{2}{|c|}{ VARIABLES EN EL MODELO } & $\begin{array}{c}\text { TOTAL } \\
\text { MUESTRA }\end{array}$ & $\begin{array}{c}\text { Q1. } \\
\text { SIN } \\
\text { PROBLEMAS }\end{array}$ & $\begin{array}{c}\text { Q2. } \\
\text { PROBLEMAS } \\
\text { TÉCNICOS }\end{array}$ & $\begin{array}{c}\text { Q3. } \\
\text { PROBLEMAS } \\
\text { PSICOSOCIALES }\end{array}$ & $\begin{array}{c}\text { Q4. } \\
\text { PROBLEMAS } \\
\text { COMPETENCIAS }\end{array}$ \\
\hline \multirow{5}{*}{$\begin{array}{l}\text { Dificultades para } \\
\text { continuar con los } \\
\text { estudios* }\end{array}$} & Perfectamente & 26,5 & 49,9 & 0,0 & 0,0 & 0,0 \\
\hline & Bien, con algunas dificultades & 50,9 & 38,1 & 73,5 & 69,6 & 63,1 \\
\hline & Con bastantes dificultades & 20,1 & 9,7 & 23,5 & 25,0 & 35,0 \\
\hline & Me ha sido casi imposible & 2,3 & 2,0 & 2,9 & 5,4 & 1,8 \\
\hline & NS/NC & 0,2 & 0,3 & 0,0 & 0,0 & 0,0 \\
\hline \multirow{4}{*}{$\begin{array}{l}\text { Consecuencias } \\
\text { confinamiento* }\end{array}$} & Sí, para bien & 23,6 & 30,1 & 29,4 & 17,9 & 13,8 \\
\hline & Sí, para mal & 44,7 & 28,9 & 50,0 & 58,9 & 65,4 \\
\hline & No & 25,9 & 36,1 & 14,7 & 14,3 & 14,3 \\
\hline & NS/NC & 5,8 & 4,9 & 5,9 & 8,9 & 6,5 \\
\hline \multirow{6}{*}{$\begin{array}{l}\text { Cumplimiento } \\
\text { de objetivos } \\
\text { académicos* }\end{array}$} & Sí, en su totalidad & 28,2 & 40,1 & 26,5 & 12,5 & 13,4 \\
\hline & Sí, casi todos & 41,2 & 35,8 & 35,3 & 46,4 & 49,3 \\
\hline & Sólo en parte & 19,5 & 14,9 & 26,5 & 33,9 & 22,1 \\
\hline & Pocos & 6,6 & 5,2 & 2,9 & 3,6 & 10,1 \\
\hline & Ninguno o casi ninguno & 2,9 & 2,0 & 5,9 & 1,8 & 4,1 \\
\hline & NS/NC & 1,7 & 2,0 & 2,9 & 1,8 & 0,9 \\
\hline
\end{tabular}

Base: jóvenes entre 15 y 29 años que han podido continuar sus estudios durante el confinamiento ( $\mathrm{N}=656$ casos).

$*$ Diferencias estadísticamente significativas al $95,5 \%$.

Q1. Sin problemas importantes. Se trata del grupo más numeroso de alumnos, puesto que representan a algo más de la mitad de los jóvenes que han podido seguir las clases online (53,2\%). Se caracterizan por puntuar por debajo del promedio tanto en el número de dificultades mencionadas como en los distintos factores que agrupan el tipo de dificultades. Se trata del grupo que menos dificultades ha experimentado (la mitad señalan haber seguido perfectamente la educación online) y a quienes menos les ha afectado el confinamiento, en sus estudios, para mal (28,9\% frente al 44,7\% global). Además, su nivel de cumplimiento de objetivos académicos también es superior al resto $75,9 \%$ han cumplido todos o casi todos, frente al 69,4\% global). Con respecto a su perfil sociodemográfico, entre los jóvenes que han tenido menos problemas durante el confinamiento hay una mayor presencia de hombres (aunque la diferencia es muy pequeña), jóvenes entre 15 y 19 años, de clase media-alta y alta y cursando educación secundaria.

Q2. Problemas técnicos. Se trata del grupo más reducido de alumnos (5,2\%), vinculado específicamente con los problemas de acceso a la red (falta de equipos, conectividad), así como con la mala organización de la educación online (ratio de alumnos excesivamente elevada, imposibilidad de realizar ciertas tareas a distancia). La práctica mayoría ha tenido algunos o bastantes problemas para continuar sus estudios: en la mitad de los casos se señala que el confinamiento les ha afectado en los estudios para mal y el grado de cumplimiento de objetivos académicos es del 61,8\% (frente al 69,4\% global). Con respecto al perfil sociodemográfico, entre aquellos jóvenes que presentan más problemas de tipo técnico destacan comparativamente los hombres (muy ligeramente), los jóvenes entre 15 y 24 años, de clase media y cursando estudios universitarios.

Q3. Problemas psico-sociales. Suponen el 8,5\% del alumnado y se vinculan especialmente con dificultades de tipo anímico y emocional, así como con la falta de tiempo para dedicar a la educación digital y la necesidad de cuidar a otras personas. Un 94,6\% mencionan haber experimentado dificultades para continuar sus estudios durante el confinamiento, un 58,9\% destaca haberse visto afectado en su curso académico para mal y se trata del grupo que peor ha cumplido los objetivos académicos: solo 
los han cumplido (en su totalidad o casi todos) el 58,9\%, frente al 69,4\% global. En cuanto al perfil sociodemográfico, entre quienes han sufrido más problemas de tipo psico-social y anímico destacan las mujeres, los mayores de 25 años, las clases bajas y media-baja, quienes estudian y trabajan a la vez.

Q4. Problemas de competencias. Suponen un tercio del alumnado $(33,1 \%)$ y sus dificultades tienen que ver con su falta de competencias para realizar las tareas (dudas, desconocimiento de cómo hacer las tareas, etc.). A su vez, conviene enfatizar que los problemas vinculados a las destrezas digitales no tienen relación únicamente con las competencias propias sino con las de la comunidad educativa. Como exploraremos más adelante, los y las jóvenes han sido especialmente críticos con el profesorado. Casi todos han experimentado dificultades con sus estudios (98,1\%), a dos terceras partes les ha afectado el confinamiento para mal, a nivel académico, y su nivel de cumplimiento de objetivos es también menor a la media (62,7\% frente al 69,4\% global). Con respecto al perfil sociodemográfico, destacan las mujeres, los jóvenes entre 20 y 24 años, quienes únicamente estudian, quienes cursan estudios universitarios.

\section{Gráfico 6. Análisis discriminante. Mapa de grupos combinados (FD1 x FD2)}
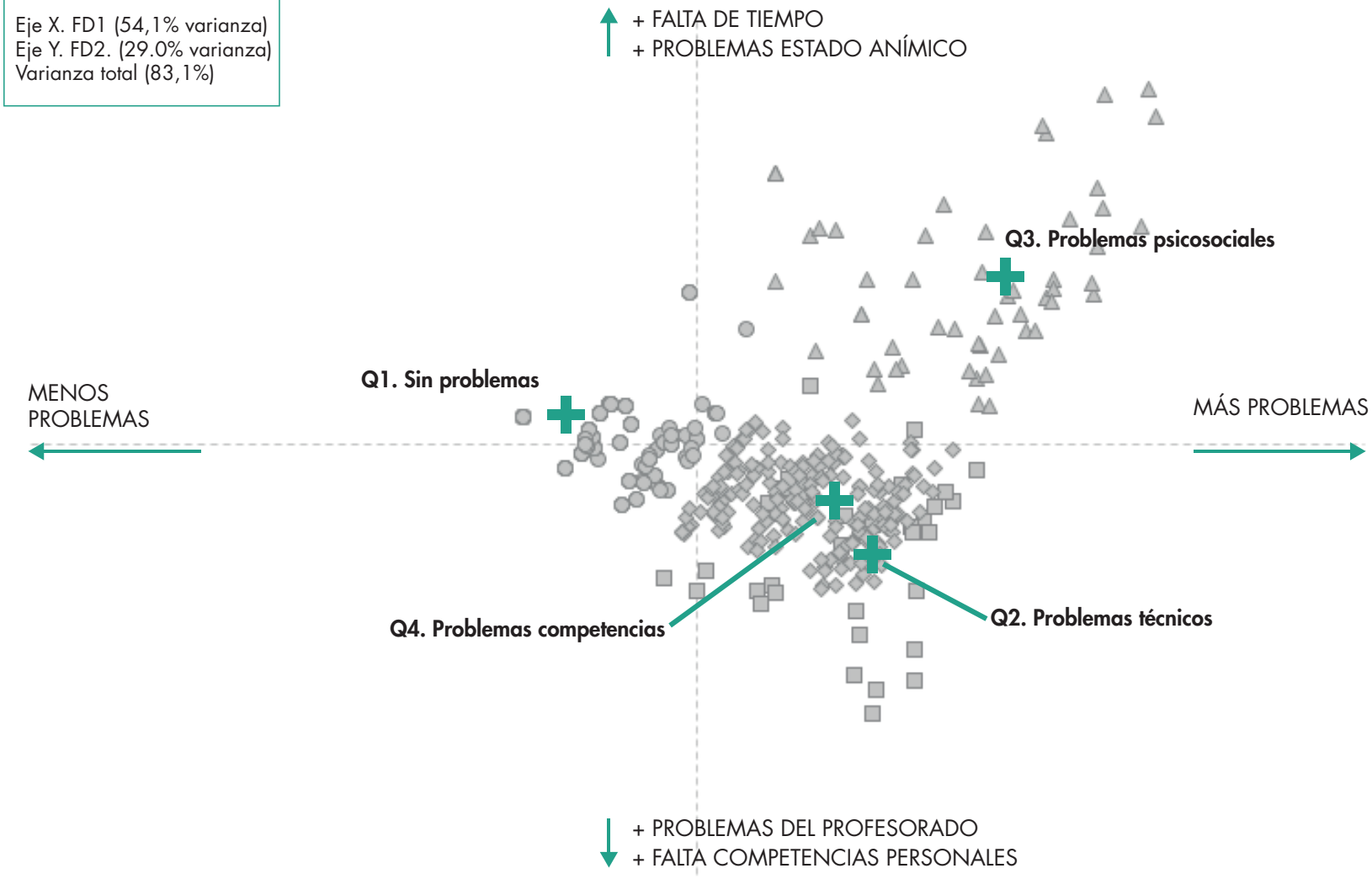

Base: jóvenes entre 15 y 29 años que han podido continuar sus estudios durante el confinamiento ( $N=656$ casos).

A partir de los resultados del análisis discriminante (Gráfico 6) es posible realizar una lectura más profunda de los datos, puesto que podemos posicionar las puntuaciones discriminantes de los casos, agrupados en base al conglomerado de pertenencia, a partir de las dos funciones discriminantes principales (FD1 en el eje de abscisas y FD2 en el de ordenadas), que acumulan el 83,1\% de la varianza del modelo. Así, vemos como el eje X, que acumula más de la mitad de la varianza, se estructura principal- 
mente a partir del total de dificultades mencionadas por los encuestados: a la izquierda encontramos al grupo Q1, que agrupa a algo más de la mitad de jóvenes (52,3\%), quienes se caracterizan por un seguimiento satisfactorio del curso académico y, a su vez, con una mayor presencia de jóvenes estudiantes de secundaria (por lo que tienen entre 15 y 29 años mayoritariamente), pero también de clase social alta y media alta. De este modo, el seguimiento del curso ha generado menos problemas en el ámbito de la educación secundaria, si bien existe un importante sesgo de clase que ha afectad mayoritariamente a las clases más bajas y colectivos más vulnerables de jóvenes, quienes se agrupan más frecuentemente en los tres conglomerados que sí han experimentado dificultades académicas durante la crisis del coronavirus (a la derecha del gráfico).

Estos tres grupos (Q2, Q3 y Q4) constituyen en conjunto el 47,7\% de los casos y se posicionan en la parte derecha del mapa, si bien muestran elementos distintivos según el tipo de dificultades vividas, motivo por el cuál es relevante el análisis en función del eje de ordenadas. De este modo, en la parte superior se posiciona claramente el grupo Q3, que supone únicamente el 8,2\% de los casos pero que se vinculan distintivamente con problemas de tipo psico-social y personal, principalmente con la falta de ánimo y la falta de tiempo para poder continuar las clases. En la parte inferior, por el contrario, encontramos posicionadas las dificultades que tienen más que ver con los problemas de competencias (tanto del alumnado como del profesorado) y de tipo organizativo: así, se posicionan en la parte inferior del gráfico los grupos Q2 (problemas técnicos) y Q4 (problemas de competencias), si bien la presencia de los problemas técnicos de accesibilidad a las herramientas tecnológicas es muy minoritaria (5,2\% de los casos) en comparación con la falta de competencias del alumnado, las dudas surgidas durante el proceso de adaptación a la educación telemática y, también, la falta de organización por parte del profesorado $(33,1 \%$ de los casos).

\section{Discusión}

En este artículo hemos analizado cómo el contexto de pandemia global ha afectado directamente la experiencia de los y las jóvenes en relación al sistema educativo, una realidad que ya ha sido constatada en varias investigaciones (Expósito y Marsollier, 2020; Penna Tosso et al., 2020; Robinson et al., 2020; Trujillo Sáez et al., 2020). A pesar de su orientación descriptiva y exploratoria, se ha dado respuesta a dos objetivos complementarios: por un lado, dimensionar el efecto de las brechas digitales y las desigualdades sociales en el seguimiento de la educación a distancia, en especial entre los grupos más vulnerables; por otro lado, establecer una tipología a partir de las problemáticas y dificultades percibidas por los alumnos en la continuación de sus estudios, que permita complementar el trabajo de Trujillo Sáez et al., (2020), centrado en la visión de la comunidad educativa, atendiendo a las necesidades específicas y diversas del alumnado. En este sentido, podemos destacar tres elementos fundamentales que marcan el camino hacia la implantación de la educación online, atendiendo a las desigualdades sociales y digitales presentes entre el alumnado.

\subsection{Primer elemento. La educación online agudiza la desigualdad}

El primer elemento a destacar es que, centrándonos en el ámbito de la educación y en los datos con los que contamos, no es sorprendente constatar que la población de clase baja y los colectivos más vulnerables se encuentran en una posición estructural significativamente más complicada a la hora de afrontar los retos que plantea el sistema educativo (Bourdieu y Passeron, 1996), agravándose ahora con su virtualización. Por un lado, es cierto que el seguimiento del curso académico durante el confi- 
namiento ha sido bastante generalizado ( 9 de cada diez alumnos continuaron las clases), siendo más relevantes las diferencias por nacionalidad (el seguimiento entre jóvenes extranjeros y de nacionalidad española adquirida) que por clase social, si bien el grupo con mayor tasa de seguimiento es la clase media. Por otro lado, un 44,7\% del alumnado destaca que la pandemia ha afectado a sus estudios para mal y el nivel de cumplimiento de los objetivos académicos está en torno al 69,4\%. En este punto sí que encontramos diferencias por clase social, de forma que hasta un 49,3\% de la población joven de clase baja-media baja afirma que el confinamiento ha afectado negativamente a su rendimiento escolar frente al 38\% de la de clase media-alta y alta. En esta línea, únicamente el 21,7\% de los jóvenes de clase baja-media baja afirma haber seguido perfectamente el curso frente al 36,4\% de los y las jóvenes de clase alta-media alta.

En cuanto a la tipología de problemáticas para continuar los estudios, algo más de la mitad de los jóvenes $(52,3 \%)$ no ha experimentado problemas importantes en el seguimiento de la educación online, destacando el alumnado más joven (15 a 19 años) y de clase media-alta y alta. Los problemas de competencias digitales propias o del profesorado destacan entre un tercio del alumnado (33,1\%), siendo significativamente mayor la presencia de mujeres y estudiantes de nivel universitario. Se trata del tipo de competencias orientadas al contenido. Por el contrario, los problemas técnicos de acceso a la tecnología y organizativos son muy poco frecuentes (5,6\%), mostrándonos como, en general, la gran mayoría de los y las jóvenes está habituada al uso de las TIC. Este resultado no es sorprendente, puesto que los niveles de digitalización, accesibilidad digital y frecuencia de uso de las TIC en España han estado tradicionalmente por encima de otros países del entorno mediterráneo y de Europa del Este, pero por detrás de los países de centro y norte de Europa (Calderón Gómez, 2019).

En este sentido, si seguimos la clasificación establecida por Van Deursen y Van Dijk (2014) entre competencias orientadas al medio (manejo técnico de los dispositivos) y orientadas al contenido (aprovechamiento digital en términos de beneficios tangibles), los resultados muestran como entre al alumnado español las dificultades han tenido más que ver con la instrumentalización de las TIC para fines educativos que con las destrezas técnicas necesarias para manejar los dispositivos, y mucho menos con el acceso material a los equipos TIC. Nos encontramos ante un problema de tercera brecha digital (Ragnedda, 2017; Van Deursen y Helsper, 2015) relacionado con el aprovechamiento de la tecnología, que ha afectado especialmente durante la pandemia a los colectivos más vulnerables y podría polarizar la desigualdad social en el futuro.

Finalmente, los problemas psicosociales (estado anímico, cuidado de otras personas, falta de tiempo) suponen únicamente el 8,5\% de los casos. Se trata del grupo con peor cumplimiento de objetivos académicos durante la pandemia y entre quienes encontramos las porciones sociales más vulnerables, destacando el alumnado de clase baja y quienes deben estudiar y trabajar a la vez, además de las mujeres y el alumnado mayor de 25 años. Nuestro despliegue metodológico únicamente nos permite dimensionar el problema; no obstante, otras investigaciones basadas en estudios de caso sí nos pueden ayudar a profundizar sobre las experiencias del alumnado que encaja en este perfil. Por un lado, investigaciones como la de Sánchez-Gelabert et al. (Sanchez-Gelabert et al., 2017) o la de Applegate y Daly (2006) permiten explorar el efecto de estudiar y trabajar de forma simultánea como respuesta a una situación de precariedad. Los efectos sobre el rendimiento académico varían en función de la intensidad del trabajo, e incluso pueden constatarse efectos positivos en algunos casos; no obstante, la mayoría 
de casos muestran un empeoramiento del rendimiento. Por otro lado, el estudio de caso analizado por González Monteagudo (2010) aborda la experiencia de estudiantes universitarios inmigrantes, con dificultades socioeconómicas, con cargas familiares o discapacidad. Esta perspectiva nos puede ayudar a analizar los retos que afronta el alumnado que encaja en el perfil con problemas psicosociales. Además, es importante destacar el impacto del género en estos perfiles, siendo las mujeres las que con más frecuencia se enfrentan a este tipo de problemáticas, como evidencia el Barómetro sobre Salud y Bienestar del CRS (Ballesteros et al., 2020). Los datos muestran que los niveles de estrés vinculados a variables como el trabajo y los estudios, las relaciones familiares e interpersonales, la situación económica y el estado mental son significativamente superiores en las mujeres que en sus pares varones (Ballesteros et al., 2020: 53).

\subsection{Segundo elemento. La educación virtual como reto del profesorado}

Un segundo elemento que queremos destacar de los datos de la investigación es el cuestionamiento del profesorado por parte de los y las estudiantes a la hora de abordar la problemática de la educación online. Un 31,4\% afirma que el profesorado no estaba bien preparado para impartir clases online y hasta un $20,4 \%$ defiende que sus profesores/as no estaban lo suficientemente pendientes. De este modo, los problemas con el profesorado se convierten en el segundo origen más frecuente de las dificultades experimentadas con la educación durante el confinamiento.

Para profundizar en esta problemática es importante tener en cuenta la evolución de la posición social del profesorado. Lejos queda la imagen del profesor como intelectual ilustrado del siglo XIx y xx, intérprete de la razón y transmisor de los hábitos de las culturas dominantes. La democratización de todos los niveles educativos a lo largo del siglo xx ha contribuido a la desacralización del conocimiento como bien escaso y ha destituido, en cierta medida, al profesorado de la posición de veneración, reconocimiento o incluso idealización que ocupaba anteriormente (Guerrero Serón, 2007). Actualmente, la autoridad del profesorado traslada buena parte de su peso de la esfera de los conocimientos sobre métodos pedagógicos. De este modo, la crucial dimensión socializadora de la pedagogía permite paliar los efectos de la erosión de su autoridad. No obstante, en un contexto de educación a distancia como el que se vivió durante el confinamiento, las competencias pedagógicas del profesorado se vieron inevitablemente limitadas ante el reto de aprender y enseñar en un entorno virtual.

La posibilidad para generar una docencia de calidad durante el confinamiento pasaba por la improvisación de metodologías nunca antes desplegadas en el global del sistema educativo español y, consecuentemente, se ha producido un desgaste muy importante de la imagen docente. Esto se ha unido al hecho de que el propio profesorado se vio obligado a compaginar las tareas docentes con urgencias domésticas cotidianas, configurando un escenario de precariedad general. A pesar del esfuerzo colectivo de la profesión y de su toma de conciencia como últimos garantes de la igualdad de oportunidades para el alumnado, la falta de recursos y plataformas adecuadas y la escasa formación para la educación a distancia tanto para alumnos como para profesores ha hecho imposible abarcar la complejidad de la situación (Trujillo Sáez et al., 2020). Además, la única posibilidad para implementar un aprendizaje en línea masivo y de calidad es contar con una estrategia coordinada por parte de la Administración para localizar las brechas digitales existentes, adaptar las infraestructuras y generar los bancos de recursos necesarios para la comunidad educativa. El programa School's out, but class's on, implementado en China 
desde el inicio de la pandemia (Zhou et al., 2020); o la guía de la OCDE A framework to guide an education response to the COVID-19 Pandemic of 2020 (Reimers y Schleicher, 2020), serían ejemplos de cómo abordar la problemática desde las administraciones. En España, sin embargo, la educación virtual corre el riesgo de desautorizar aún más a los claustros al convertir la educación en un sistema tecnificado o instrumental de entrega de conocimientos (Giroux et al., 2020). Como analiza Klees (2020), en un contexto capitalista como el actual, los retos y problemas de los sistemas educativos tienden a atribuirse a la falta de capacidades individuales del alumnado o del profesorado en lugar de poner el foco sobre la composición y los recursos estructurales con los que cuentan los centros educativos. Esto difumina la raíz del problema y ha dificultado el abordaje de la educación online desde un análisis de las diferencias de recursos y necesidades en cada contexto escolar. Los resultados que hemos ido desplegando a lo largo del texto pueden ser un reflejo de esta realidad.

\subsection{Tercer elemento. El nivel educativo como factor significativo}

El tercer ámbito en el que queremos incidir es sobre las diferencias que observamos entre estudiantes de secundaria y estudiantes universitarios. La literatura nos muestra que un mayor nivel educativo tiende a venir asociado con un desarrollo superior de la alfabetización digital, si bien gran parte de los procesos de alfabetización se producen de manera informal en las propias prácticas cotidianas de apropiación de las tecnologías (Calderón Gómez, 2020; Robinson, 2009; Sefton-Green et al., 2009). Se desarrollan competencias que permiten ampliar el capital digital, como la habilidad para buscar información, frente a otras actividades más frecuentes entre personas con niveles educativos inferiores que no mejoran necesariamente las habilidades o el conocimiento sobre el entorno digital, como es el consumo pasivo de contenido audiovisual (Hargittai y Hinnant, 2008; Robinson y Schulz, 2013: 543).

Basándonos en esto, podríamos asumir que los estudiantes universitarios, al contar con un mayor nivel educativo, también cuentan con la capacidad para gestionar las necesidades ligadas a la educación online con mayor solvencia que estudiantes de secundaria. No obstante, los datos nos muestran la tendencia contraria: Sólo un $23.9 \%$ de estudiantes universitarios consideran que han resuelto todos sus objetivos académicos frente al 32,9\% de estudiantes de secundaria. En la misma línea, el promedio de los problemas observados por la población universitaria es de 2,28, frente al 1,72 de los estudiantes de secundaria.

La vocación de este texto es descriptiva y para poder profundizar sobre estas diferencias sería necesario desplegar aproximaciones metodológicas específicas; sin embargo, podemos introducir tres hipótesis para ayudar a organizar el debate en futuras inserciones sobre el tema. La primera es que el sistema universitario español ha sido menos maleable que los centros educativos de secundaria y ha optado por paralizar las clases antes que adaptarse a una educación online. La segunda es que la población universitaria, aunque cuenta con mejores competencias digitales, también tiene expectativas más exigentes sobre su formación que los llevan a ser más críticos ante el contexto y las faltas del panorama actual. Esta segunda hipótesis estaría apoyada por los datos del Barómetro de Autonomía Económica y Emancipación del CRS, que muestran cómo solo 2 de cada 10 jóvenes (un 23,5\%) considera que la formación universitaria está adaptada al actual sistema laboral (Tudela et al., 2020). Por último, una tercera hipótesis se podría articular analizando los diferentes niveles de exigencia en los itinerarios curriculares según la etapa educativa diferenciando entre la educación obligatoria y la postobligatoria 
puesto que analizar los períodos de transición educativa son fundamentales a la hora de dimensionar el impacto de las desigualdades sociales (Tarabini, 2020). Los resultados obtenidos únicamente nos permiten aventurar estas tres hipótesis, no obstante, la falta de una muestra más amplia impide profundizar sobre las mismas. Futuras investigaciones sobre el tema requerirían un foco más específico en este sentido.

\section{Conclusión}

El conocimiento estadístico sobre los determinantes sociales del fracaso escolar resulta de gran ayuda a la hora de analizar las realidades escolares de cerca, como lugar de acciones e interacciones múltiples (Lahire, 2008: 58). Una vez constatados los datos de esta investigación, convendría desplegar mecanismos metodológicos cualitativos que permitieran analizar las experiencias y la realidad particular en la que viven estos estudiantes. En cualquier caso, el freno de la actividad presencial en los centros educativos ha iluminado las desigualdades sociales preexistentes y nos ha planteado el reto de reestructurar colectivamente los sistemas pedagógicos para que la segregación no vaya en aumento (Giroux et al., 2020). Como destaca Martín Criado (2018), la retórica sobre los problemas educativos suelen tener como punto ciego la desigualdad de clases, de forma que suele culpabilizar al alumnado de clase baja de falta de voluntad y esfuerzo, así como al profesorado de falta de motivación, mientras se invisibilizan los problemas estructurales y se desmantela el sistema público de educación.

De este modo, la desigualdad a la hora de afrontar retos académicos es un elemento estructural previo a la pandemia, pero la situación de confinamiento ha intensificado muchas de las dificultades experimentadas por el alumnado se han visto intensificadas. Algunos ejemplos se pueden concretar en el incremento de cargas familiares fruto de la reorganización de los modelos de convivencia con el teletrabajo y el telestudio, la aparición de nuevas tareas de cuidados para suplir a las personas de mayor edad — con perfiles de riesgo- en su función de garantes de la estabilidad familiar (Bloise y Aguado-Bloise, 2019), o la pérdida de poder adquisitivo ante la destrucción masiva de empleo juvenil.

Por otro lado, el cuestionamiento del profesorado se ha convertido en una salida frecuente por parte del alumnado a la hora de justificar las problemáticas a las que se enfrentan. Sin embargo, podemos plantear la hipótesis de que también se está produciendo el efecto contrario, una revalorización de la educación tanto presencial como telemática y de la figura del profesor o profesora como garante de una educación de calidad. Esto también nos podría ayudar a explicar el posicionamiento especialmente crítico del alumnado universitario. Todavía es pronto para pronosticar la evolución del sistema educativo, pero resulta evidente que las carencias enfatizadas por el contexto actual deben formar parte de los debates entre los agentes implicados en su diseño: alumnado, familias y asociaciones de AMPAS, profesorado y perfiles profesionales técnicos y políticos.

\section{Referencias bibliográficas}

Applegate, Craig y Daly, Anne (2006): "The Impact of Paid Work on the Academic Performance of Students: A Case Study from the University of Canberra”. Australian Journal of Education, 50, 155-166.

Ballesteros, Juan Carlos; Sanmartín, Anna; Tudela, Patricia y Rubio, Ana (2020). Barómetro juvenil sobre Saludy Bienestar 2019. Madrid: Centro Reina Sofía sobre Adolescencia y Juventud, Fad. DOI: https:// doi.org/10.5281/zenodo.3768432. 
Bloise, E. A., y Aguado-Bloise, Empar (2019). Mujeres y hombres frente al desempleo. El caso español en la primera crisis del siglo XXI. Valencia: Tirant Humanidades. Quaderns Feministes.

Bonal, Xavier y González, Sheila (2020). "The Impact of Lockdown on the Learning Gap: Family and School Divisions in Times of Crisis". International Review of Education. DOI: https://doi.org/10.1007/ s11159-020-09860-z.

Calderón Gómez, Daniel (2019). Capital digital y socialización tecnológica: una aproximación bourdiana al estudio de la desigualdad digital y la estratificación social entre la juventud (Tesis doctoral). Universidad Complutense de Madrid. Disponible en https://eprints.ucm.es/58013/.

Calderón Gómez, Daniel (2020): “Technological Socialization and Digital Inclusion: Understanding Digital Literacy Biographies among Young People in Madrid”. Social Inclusion, 8 (2), 222-232. DOI: https://doi.org/10.17645/si.v8i2.2601.

Castaño, Cecilia (2008). La segunda brecha digital. Madrid: Cátedra.

Castells, Manuel (2011). La era de la información volumen 1: La sociedad red. Madrid: Alianza Editorial.

Cea D’Ancona, María Ángeles (2016). Análisis discriminante. Madrid: Centro de Investigaciones Sociológicas.

Díez, Rubén; Belli, Simone y Márquez, Israel V. (2020): "La COVID-19, pantallas y reflexividad social. Cómo el brote de un patógeno está afectando nuestra cotidianidad". Revista Española de Sociología, 29 (3), 759-768. DOI: https://doi.org/10.22325/fes/res.2020.49.

Expósito, Cristián David y Marsollier, Roxana Graciela (2020): "Virtualidad y educación en tiempos de COVID-19. Un estudio empírico en Argentina”. Educación y Humanismo, 22 (39), 1-22. DOI: https:/ / doi.org/https://doi.org/10.17081/eduhum.22.39.4214.

Feather, John (2013). The Information Society: A Study of Continuity and Change. London: Facet Publishing.

Fundación ANAR. (2020). Fundación ANAR lleva al Congreso la violencia a la infancia y adolescencia durante la pandemia COVID-19 (en línea). https://www.anar.org/fundacion-anar-congreso-violencia-infancia-adolescencia-durante-pandemia-covid19/.

Giroux, Henry; Rivera-Vargas, Pablo y Passeron, Ezequiel (2020): “Pedagogía Pandémica. Reproducción Funcional o Educación Antihegemónica”. Revista Internacional de Educación para la Justicia Social, 9 (3).

Gonzales, Amy (2016): “The Contemporary US Digital Divide: From Initial Access to Technology Maintenance". Information, Communication \& Society, 19 (2), 234-248. DOI: https://doi.org/10.1080/136911 8X.2015.1050438.

González-Monteagudo, José (2010): “Biografía, identidad y aprendizaje en estudiantes universitarios no tradicionales. Estudio de caso de una mujer trabajadora". Profesorado, Revista de Currículum y Formación de Profesorado, $14(3), 131-147$.

Guerrero Serón, Antonio (2007): “El análisis sociológico del profesorado: categoría social y agente educativo". Educación y futuro: Revista de investigación aplicada y experiencias educativas, 17 (17), 43-72. 
Hargittai, Eszter (2002): "Second-Level Digital Divide: Differences in People's Online Skills". First Monday, 7 (4), 1-14. Disponible en http:/ / firstmonday.org/article/view/942/864\#author.

Hargittai, Eszter y Hinnant, Amanda (2008). "Digital inequality: Differences in young adults' use of the Internet”. Communication Research, 35 (5), 602-621. DOI: https://doi.org/10.1177/0093650208321782.

Klees, Steven J. (2020): “Beyond Neoliberalism: Reflections on Capitalism and Education”. Policy Futures in Education, 18 (1), 9-29. DOI: https://doi.org/10.1177/1478210317715814.

Lahire, Bernard (2008): "Un sociólogo en el aula: objetos en juego y modalidades" en María Isabel Jociles y Adela Franzé Mundanó (eds.): ¿Es la escuela el problema?: perspectivas socio-antropológicas de etnografía y educación. Madrid: Trotta.

Lobera, Josep y Cabrera, Pablo (2020). Percepción social de aspectos científicos del COVID-19 (en línea). https://www.ciencia.gob.es/stfls/MICINN/Ministerio/FICHEROS/tablas_resultados_encuesta_ vacunas_1era_oleada.pdf.

Martín Criado, Enrique (2018): "Juventud y educación: cuestión de clase”. Encruaijadas. Revista crítica de Ciencias Sociales, 15 (1501), 1-17.

Nguyen, An y Catalan-Matamoros, Daniel (2020): “Digital Mis/disinformation and Public Engagment with Health and Science Controversies: Fresh Perspectives from COVID-19". Media and Communication, 8 (2), 323-328. DOI: https://doi.org/10.17645/mac.v8i2.3352.

Oltra, Christian y Boso, Àlex (2020): "Lecciones aprendidas de la crisis del coronavirus: preparación y resiliencia social”. Revista Española de Sociología, 29 (3), 769-775. DOI: https://doi.org/10.22325/fes/ res.2020.50.

Penna Tosso, Melani; Sánchez Sáinz, Mercedes y Mateos Casado, Cristina (2020): "Desigualdades educativas derivadas del COVID-19 desde una perspectiva feminista.: Análisis de los discursos de profesionales de la educación madrileña”. Revista internacional de educación para la justicia social (RIEJS), 9 (3), 157-180. Disponible en https://dialnet.unirioja.es/servlet/articulo?codigo=7523267\&orden=0\&info $=$ link \%0Ahttps: / / dialnet.unirioja.es/servlet/ extart?codigo $=7523267$.

Ragnedda, Massimo (2017). The Third Digital Divide: A Weberian Approach to Digital Inequalities. New York: Routledge.

Reimers, Fernando M. y Schleicher, Andreas (2020). A Framework to Guide an Education Response to the COVID-19 Pandemic of 2020. OECD. Disponible en https://learningportal.iiep.unesco.org/es/ node/ 73779 .

Reisdorf, Bianca y Rhinesmith, Colin (2020): "Digital Inclusion as a Core Component of Social Inclusion”. Social Inclusion, 8 (2), 132-137. DOI: https://doi.org/10.17645/si.v8i2.3184.

Robinson, Laura (2009): “A Taste for the Necessary”. Information, Communication \& Society, 12 (4), 488-507. DOI: https://doi.org/10.1080/13691180902857678.

Robinson, Laura y Schulz, Jeremy (2013): "Net Time Negotiations Within the Family". Information Communication and Society, 16 (4), 542-560. DOI: https://doi.org/10.1080/1369118X.2013.777761. 
Robinson, Laura; Schulz, Jeremy; Khilnani, Aneka; Ono, Hiroshi; Cotten, Shelia R.; McClain, Noah; Tolentino, Lloyd; Chen, Wenhong; Huang, Gejun; Casilli, Antonio A.; Tubaro, Paola; Dodel, Matías; Quan-Haase, Anabel; Ruiu, Maria Laura; Ragnedda, Massimo; Aikat, Deb y Tolentino, Natalia (2020): "Digital Inequalities in Time of Pandemic: COVID-19 Exposure Risk Profiles and New Forms of Vulnerability". First Monday, 25 (7). DOI: https://doi.org/https://doi.org/10.5210/fm.v25i7.10845.

Rubio Castillo, Ana; Sanmartín Ortí, Anna; Tudela Canaviri, Patricia; Ballesteros Guerra, Juan Carlos (2020). Barómetro general 2019: autonomía económica y emancipación. Madrid: Centro Reina Sofía sobre Adolescencia y Juventud, Fad. DOI: https://doi.org/10.5281/zenodo.3600993.

Sanchez-Gelabert, Albert; Figueroa, Mijail y Elias, Marina (2017): “Working Whilst Studying in Higher Education: The impact of the Economic Crisis on Academic and Labour Market Success". European Journal of Education, 52 (2), 232-245. DOI: https://doi.org/10.1111/ejed.12212.

Sanmartín, Anna; Ballesteros, Juan Carlos; Calderón, Daniel y Kuric, Stribor (2020). De puertas adentro y de pantallas afuera. Jóvenes en confinamiento. Madrid: Centro Reina Sofía sobre Adolescencia y Juventud, Fad. DOI: https://doi.org/10.5281/zenodo.4054836.

Sefton-Green, Julian; Nixon, Helen y Erstad, Ola (2009): "Reviewing Approaches and Perspectives on 'Digital Literacy"'. Pedagogies: An International Journal, 4(2), 107-125. DOI: https://doi. org/10.1080/15544800902741556.

Sevilla, Almudena; Sainz, Jorge y Marcen, Miriam (2020). Existen riesgos en la reapertura de los centros educativos, pero mantenerlos cerrados agrava las desigualdades de oportunidades por nivel socio-económico (en línea). https://nadaesgratis.es/admin/existen-riesgos-en-la-reapertura-de-los-centros-educativos-pero-mantenerlos-cerrados-agrava-las-desigualdades-de-oportunidades-por-nivel-socio-economico\#_ftn10.

Tarabini, Aina (2020): "Presentación: transiciones educativas y desigualdades sociales: una perspectiva sociológica”. Papers. Revista de Sociologia, 105 (2), 177. DOI: https://doi.org/10.5565/rev/papers.2825.

Trujillo Sáez, Fernando; Fernández Navas, Manuel; Montes-Rodríguez, Ramón; Segura Robles, Adrián; Alaminos Romero, Francisco J. y Postigo-Fuentes, Ana Yara (2020). Panorama de la educación en España tras la pandemia de COVID-19: la opinión de la comunidad educativa. Madrid: FAD. DOI: https://doi. org/10.5281/zenodo.3878844.

Van Deursen, Alexander (2020): "Digital Inequality During a Pandemic: Differences in COVID-19-Related Internet Uses and Outcomes among the General Population". Journal of Medical Internet Research, 22 (8), 1-13. DOI: https://doi.org/10.2196/20073.

Van Deursen, Alexander y Helsper, Ellen J. (2015): “The Third-Level Digital Divide: Who Benefits Most of Being Online?” en Laura Robinson, Shelia R. Cotten, Jeremy Schulz (eds.): Communication and Information Technologies Annual. Digital Distinctions and Inequalities (Vol. 10). Bingley: Emerald Group.

Van Deursen, Alexander y van Dijk, Jan (2019). “The First-Level Digital Divide Shifts from Inequalities in Physical Access to Inequalities in Material Access”. New Media \& Society, 21 (2), 354-375. DOI: https://doi.org/10.1177/1461444818797082. 
Van Deursen, Alexander y van Dijk, Jan (2014). Digital Skills. Unlocking the Information Society. Palgrave Macmillan. DOI: https://doi.org/10.1057/9781137437037.

Zhou, Longjun; Li, Fangmei ; Wu, Shanshan y Zhou, Ming (2020). 'School's Out, But Class' On, The Largest Online Education in the World Today: Taking China's Practical Exploration During The COVID-19 Epidemic Prevention and Control As an Example". Best Evidence Chinese Education, 4 (2), 501-519. DOI: https://doi.org/10.15354/bece.20.ar023.

\section{Notas biográficas}

Stribor Kuric Kardelis es técnico de Investigación Social en el Centro Reina Sofía de FAD sobre Adolescencia y Juventud. Doctor en Sociología y Antropología por la UCM, sus principales líneas de investigación son la sociología del trabajo y la sociología de la cultura y el arte. Especializado en metodologías de investigación cualitativas y participativas.

Daniel Calderón Gómez es técnico de Investigación Social en el Centro Reina Sofía de FAD sobre Adolescencia y Juventud. Doctor en Sociología y Antropología por la UCM, sus principales líneas de investigación son la sociología de la juventud, la sociedad digital y el estudio de las desigualdades digitales. Especializado en metodologías de investigación cuantitativas.

Anna Sanmartín Ortí es subdirectora del Centro Reina Sofía sobre Adolescencia y Juventud de FAD, doctora en Sociología por la UCM y miembro de la Red de Estudios sobre Juventud y Sociedad (REJS). Se ha especializado en el análisis de la socialización juvenil, con publicaciones que estudian los roles de género, la participación política, el ocio y los consumos o el impacto de las tecnologías. 